\title{
Summary of R\&D Activities for the Production of the Divertor Target Elements of Wendelstein 7-X
}

\author{
J. Boscary, A. Peacock, M. Smirnow, H. Tittes \\ Max Planck Institute for Plasma Physics, EURATOM Association, Garching, Germany \\ Corresponding author e-mail: jean.boscary@ipp.mpg.de
}

\begin{abstract}
The realization of the high heat flux divertor of the stellarator Wendelstein $7-X$ requires the production of 890 target elements. Since the divertor has to operate for 30 minutes with an input $\mathrm{cw}$ plasma power of $10 \mathrm{MW}$, the target elements need to be actively water-cooled. A target element is made of a CuCrZr copper alloy heat sink armored with CFC NB31 tiles. It is designed to remove a stationary heat flux of $10 \mathrm{MW} / \mathrm{m}^{2}$ on its main area and $5 \mathrm{MW} / \mathrm{m}^{2}$ at the end adjacent to the pumping gap. $R \& D$ activities proved to be essential for the preparation and the support of the on-going serial fabrication. The most critical issue is the stable production of the bonding between the CFC tiles and the heat sink as the divertor surface consists of about $\mathbf{1 6 , 0 0 0}$ tiles. The fast unexpected failure of one tile may reduce the whole divertor performance and, consequently, restrict the stationary machine operation. Two main $R \& D$ phases allow the design improvements of the target elements compatible with industrial and economic constrains of the series production as well as the development of quality controls such as a visual inspection system able to easily detect and fast record cracks at the interface between CFC tiles and heat sink.
\end{abstract}

Keywords-Stellarator; Wendelstein 7-X; Plasma Facing Component; Divertor

\section{INTRODUCTION}

The stellarator Wendelstein 7-X (W7-X) is presently under completion in the Max Planck Institute of Greifswald, Germany. This experimental machine is designed to operate stationary plasma over a pulse length of up to 30 min. with a $10 \mathrm{MW}$ cw input power and peak power with additional combined heating systems of up to $24 \mathrm{MW}$ for 10s [1], [2]. Due to the particular geometry of W7-X, the divertor follows the twisted plasma shape. The $19.6 \mathrm{~m}^{2}$ area of the high heat flux divertor consists of 890 individual actively cooled target elements. After the initial commissioning phase, the divertor operation will start in 2016 with an inertially cooled divertor made of graphite tiles clamped onto a stainless steel support structure designed for 8MW cw input power for $10 \mathrm{sec}$ [3], [4]. The high heat flux divertor will be operated from 2019 on.

Before launching the production of the series target elements, extended R\&D activities divided into two main phases have been carried out. The purpose was the validation of the design, the manufacturing route, the inspection methods and facilities, and the definition of the relevant acceptance criteria taking into account the economic constrains and industrial environment of the serial production. The most critical issue to ensure the long pulse divertor operation is the bonding of the tiles to the heat sink as the divertor surface consists of about 16,000 tiles. Most R\&D activities focused on the development of this technology to reach a reliable and industrially reproducible production of this bonding because the fast unexpected failure of one tile may restrict the machine operation.

\section{DESCRIPTION OF DIVERTOR TARGET ELEMENTS}

The ten divertor discrete units, two units per field period, are placed along the twisted plasma shape. Each unit has ten high heat flux target modules, as shown in Fig. 1. Each module has a set of 8-12 target elements of the same length mounted onto a support frame. The target elements are fed with water, mostly in parallel, from manifolds.

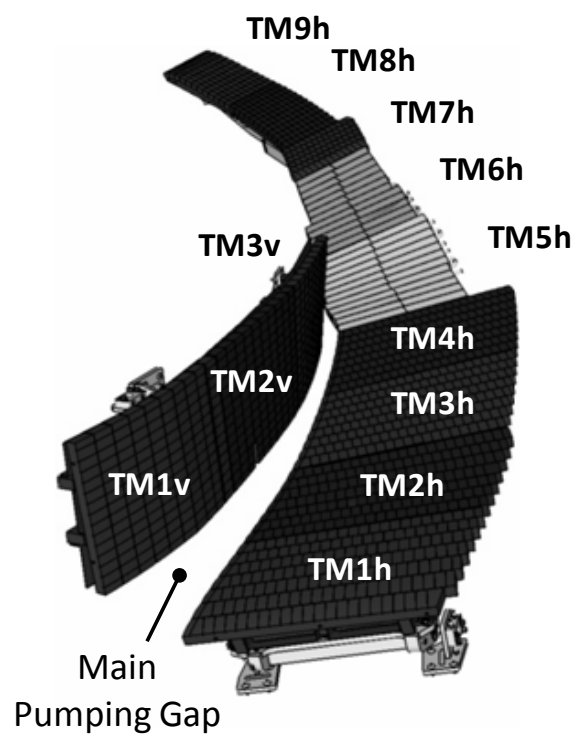

Fig. 1. Position of target module (TM) in one divertor unit (the mechanical support and water-cooling systems are partially shown).

The central part of the divertor unit (TM5h and TM6h), $0.6 \mathrm{~m}^{2}$, is designed to remove a $1 \mathrm{MW} / \mathrm{m}^{2}$ heat flux and is made of graphite tiles clamped onto a CuCrZr heat sink [5]. 
The remaining ten target modules are made of 89 target elements of five basic types and seven additional variants to integrate diagnostics.

The high heat flux divertor requires the production of 890 target elements and additional 50 spare elements will be fabricated. The distribution of the total quantity of target elements to be produced is shown in Fig. 2.

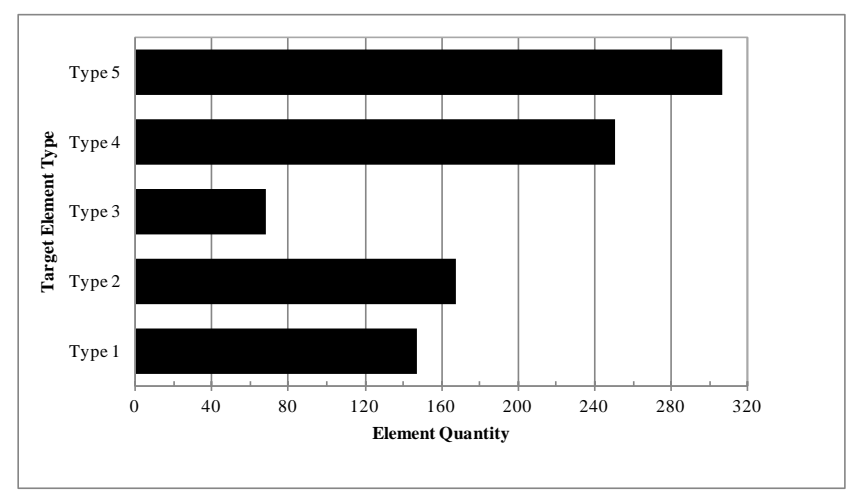

Fig. 2. Total quantity of target elements per basic type.

The distribution of the basic target element type as a function of the target module as well as the geometry of the target element is summarized in Table I.

TABLE I. DISTRIBUTION AND DIMENSIONS OF THE TARGET ELEMENTS

\begin{tabular}{|c|l|c|c|c|}
\hline $\begin{array}{c}\text { Target } \\
\text { Element } \\
\text { Type }\end{array}$ & Target Module & $\begin{array}{c}\text { Max. Length } \\
\text { [mm] }\end{array}$ & $\begin{array}{c}\text { Max. Width } \\
\text { [mm] }\end{array}$ & $\begin{array}{c}\text { Total Tile } \\
\text { Quantity }\end{array}$ \\
\hline 1 & TM01H-02H & 595 & $57-61.5$ & 25 \\
\hline 2 & TM03H-04H & 573 & $55-59.5$ & 24 \\
\hline 3 & TM07H & 320 & $54-59$ & 13 \\
\hline 4 & TM08H-09H & 250 & $57-61.5$ & 10 \\
\hline 5 & TM01V-03V & 361 & 55 & 16 \\
\hline
\end{tabular}

The target elements vary in length and width from 250 to $595 \mathrm{~mm}$ and from 50 to $61.5 \mathrm{~mm}$, respectively. The target element shape is slightly trapezoidal along and across the element. The total number of tiles per element varies from 10 to 25 tiles.

The design and technology used for the fabrication of the target elements is based on the flat tile geometry. A target element is made of water-cooled CuCrZr copper alloy heat sink armored with flat tiles made of CFC (Carbon Fiber reinforced Carbon) NB31. CFC was produced by the company Snecma Propulsion Solide located in France. A cross-section of the target element identifying the different materials is presented in Fig. 3. A $3 \mathrm{~mm}$ thick bi-layer is used for the bonding between CFC tiles and CuCrZr heat sinks. The first step is the casting of copper onto the CFC side of the tile. The second step is the joining by Hot Isostatic Pressing of an OF-copper additional layer to produce individual bi-layer tiles. The heat sinks are manufactured in parallel. The cooling channel is a meander of 4 straight parts connected by 3 bends. Semi circular channels are machined into the two half plates of the heat sink, which are then electron beam welded together. A stainless steel twisted tape, $1 \mathrm{~mm}$ thick and twist ratio 2, is placed in the straight part of the cooling channel and is mechanically attached to the heat sink by a key-slot system. This tape acts as a turbulence promoter increasing the margin to the critical heat flux. In a final stage, the CFC tiles are electron beam welded to the CuCrZr heat sink.

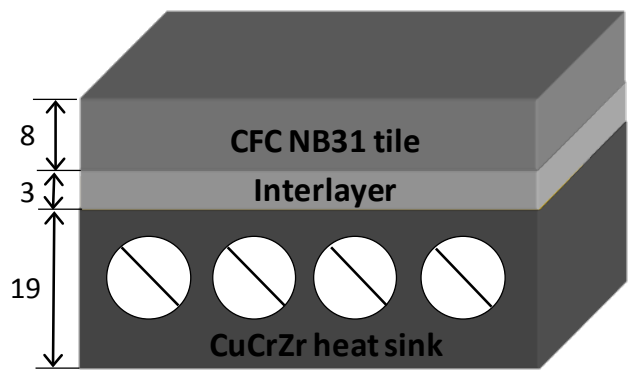

Fig. 3. Cross-section of a manufactured target element.

The arrangement and specification of the tiles depends on the position along the target element and on the target module type. The tiles covering the main central divertor surface, typically $25 \mathrm{~mm}$ long, are specified to withstand a stationary heat flux of $10 \mathrm{MW} / \mathrm{m}^{2}$. The target modules TM1-4H and TM1V-3V are adjacent to the main pumping gap. At this location, the extremity of the target element needs a special design and the specified heat load is reduced: a stationary heat flux of $5 \mathrm{MW} / \mathrm{m}^{2}$ for the tiles placed at the end of the top surface and $2 \mathrm{MW} / \mathrm{m}^{2}$ for the tiles placed at the end facing the pumping gap.

The production of the target elements has been divided into two phases: 282 elements (TM 7H, TM8H and TM9H) started in 2009 and completed in 2011, 658 elements (other target modules) started in 2011 and to be completed in 2014. Presently (June 2013), 409 elements, i.e. 43.5\% of the whole production, have been delivered. The percentage of delivered element per basic type is detailed in Fig. 4.

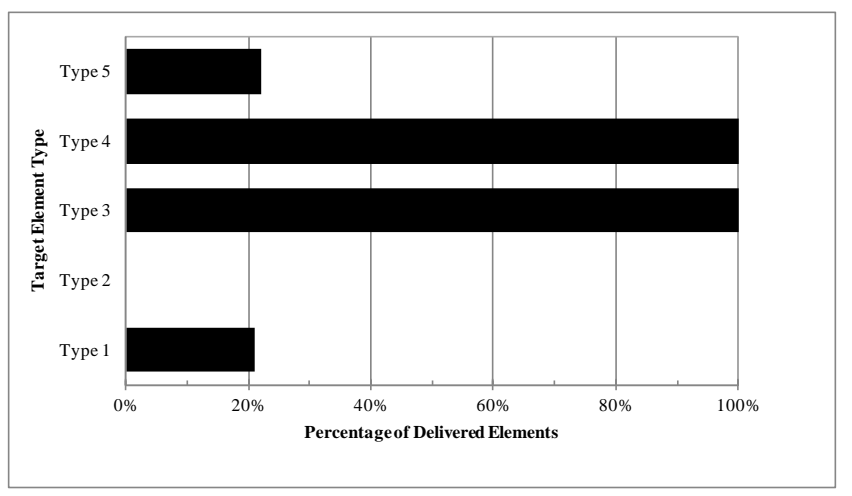

Fig. 4. Quantity of delivered target elements (Status June 2013) per basic type. 


\section{SUMMARY OF R\&D ACTIVITIES}

The initial solution was the scaling of the design from the finger elements manufactured for the tokamak Tore Supra [6] in order to minimize R\&D activities. The main differences between the toroidal pump limiter of Tore Supra and the W7-X divertor are: a quantity of 600 fingers for Tore Supra compared to 940 target elements for W7-X (+ $57 \%$ ), a total surface of $7.5 \mathrm{~m}^{2}$ for Tore Supra compared to $19.6 \mathrm{~m}^{2}$ for W7-X $(+261 \%)$. The lessons learned from the design, quality control and production of the finger elements were firstly carefully examined in collaboration with the Tore Supra team before launching the fabrication. A reliable bonding between the tiles and the heat sink [7] proved to be the most difficult issue of the manufacturing process. The same technology used for Tore Supra, i.e. Active Metal Casting, was selected and to improve its reliability, the CFC N11 used for Tore Supra was replaced by the CFC NB31, which has better mechanical properties. Developed during EFDA R\&D activities, this grade was the reference material for the ITER divertor at that time, presently replaced by CFC NB41 [8]. This decision was also supported by the results of the different high heat flux testing of prototypes validating the compatibility of this material with the AMC technology [9]. The analysis of the W7-X design also showed that the manufacturing process of the heat sink had to be modified because of the maximal length and of the geometry of the cooling channel of the target elements. Instead of drilling, the heat sink is produced by the electron beam welding of a base heat sink and a back plate into which half-channels are machined. To minimize the risks, the same grade and the same producer as Tore Supra for the CuCrZr copper alloy of the heat sink was chosen. Finally, a pre-series phase aiming at demonstrating an extremely low rate of defective tiles and a reproducibility of the element performance to guarantee possible stable and economical viable serial production was included in the contract. The Austrian company Plansee SE was selected for the production of the W7-X target elements.

\section{A. Pre-series Phase I}

The results of the first pre-series clearly demonstrated that the transfer of the experience gained in Tore Supra to W7-X by scaling was insufficient and that additional R\&D activities were required. Instead of one originally planned, a total of four pre-series were necessary before launching the fabrication [10], [11], [12], [13]. It required the fabrication and testing of 60 full-scale target elements. Since each pre-series lasted about one year including the element fabrication, testing and result assessment, a total of 4 years instead of the 1 year initially planned were needed. Additional difficulties were encountered during the CFC NB31 production. The second pre-series concentrated on the assessment of the effect of a CFC with lower mechanical properties than specified on the element performance [14].

In this process, a key tool was the possibility of testing the elements in the high heat flux facility Gladis [15] to assess the performance and the reproducibility of the proposed solutions. As a critical part of the decision process, the acceptance criteria are specified as follows: the elements must withstand 100 cycles at $10 \mathrm{MW} / \mathrm{m}^{2}$ (10 seconds loading) without showing any performance degradation such as crack initiation or propagation at the interface between tiles and heat sink.

The main achievements of this first phase can be summarized as follows:

- The development of a new metallic bonding technology of the CFC tiles, namely, the bi-layer technology.

- The selection of the electron beam welding solution to join the bi-layer tiles to the heat sinks.

- The release of the CFC NB31 batches produced for W7-X.

- The optimized welding design of the heat sink.

The conclusion of these activities was the release of the selected technology for the geometry tested: short elements ( $\leq 320 \mathrm{~mm}$ ) without end tiles facing the pumping gap and of a basic type. These target elements will be installed in the target modules TM7H-TM9H. It was also decided to undertake additional development activities required to accept the technology for the remaining element types. Consequently the serial fabrication was re-organized into two parts. In parallel to the serial fabrication of the released geometry, an additional pre-series phase II was launched.

\section{B. Pre-series Phase II}

This phase focused on improving of the design of the longest element $(>320 \mathrm{~mm}$ ) heat sink and of the end tile as well as of the incoming inspections of the bonding between the tiles and heat sink.

\section{1) Heat Sink Improvement}

The design of the cooling channel should ensure a uniform surface temperature distribution during operation, an efficient cooling at the edges of the element, and adequate water velocity at the end of the target element. The wall thickness between neighboring straight parts of the channel should be as thin as possible to achieve a uniform surface temperature during loading. The cooling of the element edges is crucial because these edges act as potential crack initiation locations. The external straight parts of the channel are parallel to the element sides to get the same distance between the coolant and the edges along the trapezoidal elements. The consequence is a variation of the wall thickness along the element between neighboring channels with the minimum value at the end facing the pumping gap. The initial design had a simple face to face contact between the two half-plates at the location of the channel. The analysis of the HHF test results of full-scale elements in Gladis showed a bypass effect between neighboring channels generating a local velocity reduction at the element end due to an insufficiently tight gap. The consequence of this leakage is the decrease of the local convective heat transfer capability inducing an increase of the interface temperature between the CFC tiles and the heat sink: reduction of the element performance, lifetime and margin to critical heat flux. To quantify this effect and to 
find an appropriate solution, additional prototypes were specially manufactured with different defect sizes at the wall between neighboring channels. The detailed study can be found in [16]. A sealing labyrinth was introduced between neighboring channels which significantly reduced bypass effects. This solution was agreed by the manufacturer and has been applied to the elements of type 1,2 and 5.

\section{2) End Tile Design}

The initial design of the end tiles was an L-shaped tile facing the pumping gap with a triangular-shaped tile as a transition to the standard rectangular top tiles. From the economical point of view, the disadvantage of this solution was the high CFC consumption to produce the L-shaped tiles. Moreover, the testing in Gladis showed the limited reliability of the neighboring triangular-shaped tile. Consequently, the design had to be modified to achieve the necessary performance and also to simplify the manufacturing process. The adopted solution is detailed in [17]. To validate the selected design, several full-scale prototypes were tested in Gladis: 5 and $2 \mathrm{MW} / \mathrm{m}^{2}$ were reliably achieved on the end top tiles and tiles facing the pumping gap, respectively.

\section{Visual examination}

The reliability of the interface between the tiles and heat sink is a major issue for the future successful steady state divertor operation. The incoming inspection of the target elements has to ensure a clear and reliable detection of possible cracks at this interface of the series element prior to the installation in the machine. This interface is controlled at different stages of the production by different nondestructive examinations: visual and X-ray inspections of the individual tiles, pulsed thermography inspection developed by the manufacturer [18]. These complementary methods act as sequential filters. This involves a significant effort due to the 16,000 tiles that need to be inspected.

Nevertheless, an inspection system independent from the manufacturer has been developed as part of the incoming inspection of the delivered elements. The method is based on the observation of the visible interface at the edges of the elements. The basic assumption is that the interface edges are representative of the bonding quality of the whole surface. Finite element simulations and experimental results showed both that thermal stresses are concentrated at the element edges under loading. At these locations, cracks can be possibly initiated [11]. The tile de-bonding is detected during high heat flux testing by a growing hot spot viewed by an infrared camera and is always correlated to visible crack in CFC at the edge. Due to the large quantity of tiles to be inspected, the purpose was to develop an automatic visual inspection system able to detect evidence of crack initiation and de-bonding. The system should also be able to store and process the generated data.

The visual inspection facility realized by the company CTMV, Germany, is shown in Fig. 5. This figure presents a long target element of type 1, $600 \mathrm{~mm}$ long element with end tiles, positioned on a stable support structure to be inspected. The support has a turning bridge allowing the sequential viewing of the two edges without removing the element from its support. On the right part of the figure, the camera with its zoom lens and the associated light system fixed on a platform which moves parallel to the element is shown. The system automation for positioning the camera and the data management is based on routines written with LabVIEW. The camera system moves sequentially along the element edge and takes pictures of the edge at each step. The geometrical parameters are the total element length and the possible variation of the tile size along the element. During the data collection, the vertical position is fixed and can be, if required, manually adjusted between two inspections.

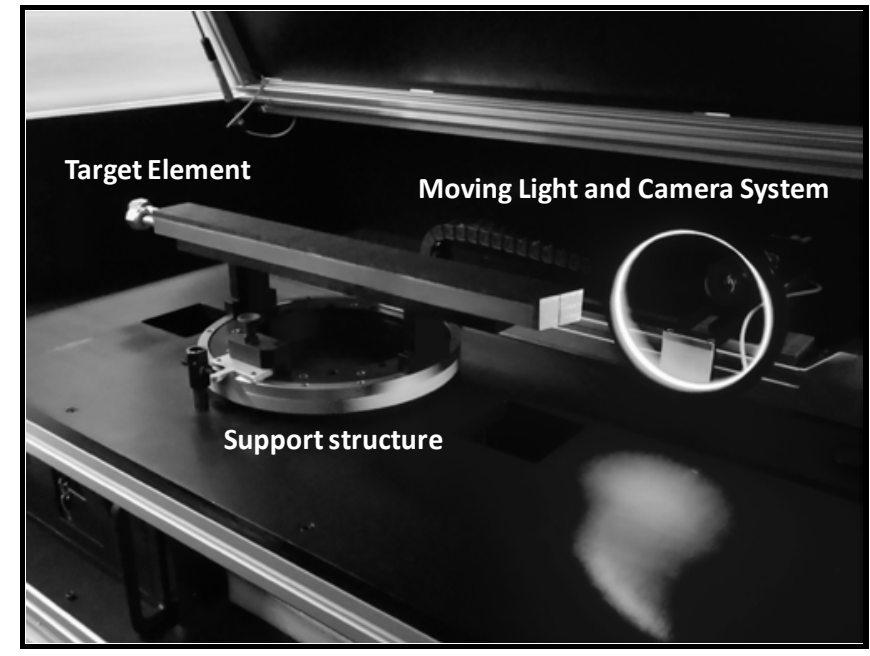

Fig. 5. Visual inspection facility.

To achieve a sufficient resolution for efficient data processing and to allow a crack to be detected, a picture of a half-tile is taken. The camera system is detailed in Fig. 6.

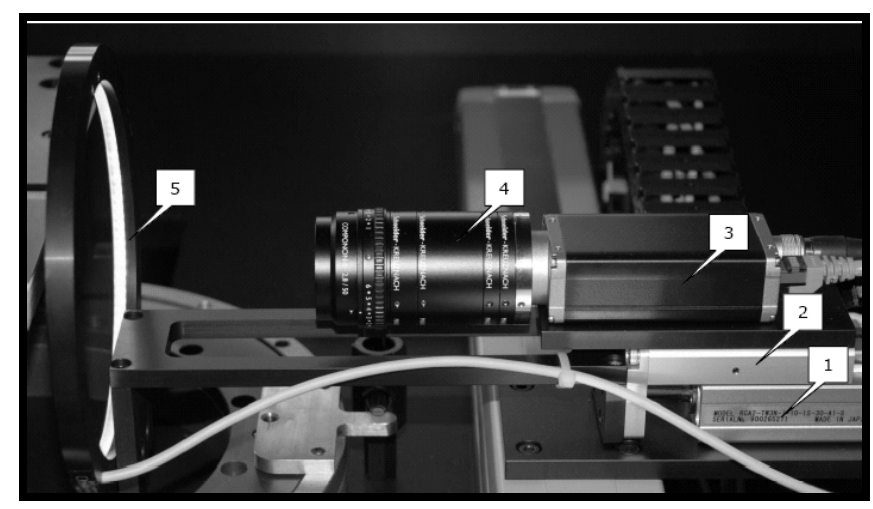

Fig. 6. Camera system of the visual inspection facility: 1,2: support axes, 3: camera, 4: zoom lens, 5: ring-light system.

The camera is a Basler type piA2400-17gm and the compact zoom lens a Schneider Kreuznach type CompononS 2.8/50. The support system allows the automatic adjustment of the distance to the element edge and a perfect focus. This distance varies between the two ends of the element due to the trapezoidal element shape. Before taking 
pictures, the camera moves to the first and to the last tile and the position of the camera for all the tiles is adjusted as an average of these two values. Once these are defined, the system takes and stores automatically the pictures of the edges.

The development of the lighting system was a major issue. The interface to be inspected is located between two materials with significantly different optical properties: dark CFC with a non-uniform surface mixture of graphite and carbon fibers absorbing the light and bright copper infiltrated in the CFC reflecting the light. The selected solution was a blue ring light LED-system surrounding the camera lens and coupled to the camera support indirectly lighting the element interface. It provides sufficient contrast to identify a crack that can be further software-analyzed. The pictures taken are black \& white. Cracks are detected by scanning the interface zone and analyzing the grey scale with a histogram. The software is very flexible and allows the operator to change the system parameters.

Fig. 7 illustrates a typical image of a half-tile with a large visible propagated crack at the interface between copper and CFC. CFC is in the upper part of the picture and on the left side the slit between two neighboring tile along the element is visible. This crack has been generated by heavily loading a full-scale pre-series element in Gladis.

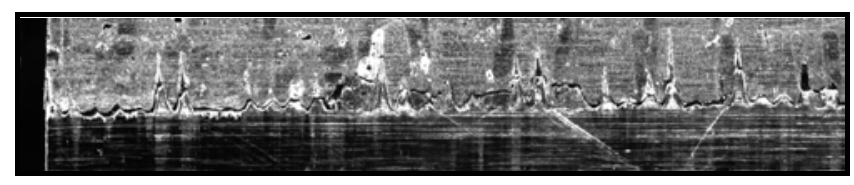

Fig. 7. Example of a large propagated crack in CFC at the interface tile heat sink.

The crack appears to have initiated at the edge (left side, slit location) and propagated in CFC along (visible) and also naturally across the interface (confirmed by metallographic examination).

The pre-series phases allowed the visual inspection system to be calibrated and validated for a reliable incoming inspection of the series target elements. Based on this experience, the acceptance criterion per tile edge is defined as follows: continuous cracks $<3 \mathrm{~mm}$ or non-discontinuous crack $<6 \mathrm{~mm}$ are accepted. The inspection results of the presently delivered series elements with this facility showed no defective tiles: 5,000 tiles, that is to say, 10,000 edges or 20,000 pictures have been recorded so far.

\section{CONCLUSION}

The actively water-cooled target elements of the W7-X high heat flux divertor are made of CFC NB31 tiles bonded onto a CuCrZr alloy heat sink. They are the highest stationary loaded W7-X plasma facing components designed to withstand a heat flux of $10 \mathrm{MW} / \mathrm{m}^{2}$ on its main area and $5 \mathrm{MW} / \mathrm{m}^{2}$ at the end defining the pumping gap.

The experience gained in the production of these elements clearly demonstrates that $R \& D$ activities must be integrated into the procurement contract before launching the industrial production of such components. A high level of collaboration with the manufacturer is mandatory to develop a common understanding of the issues that need to be addressed at an early stage of the industrial production; otherwise economic constraints become difficult to address. A clear definition of the acceptance criteria at the different steps of the production and for the incoming inspections of the delivered elements is also needed.

The main outcomes were the development of the bilayer technology, the design improvements of the heat sink and end tiles, and the development of the visual inspection system for the interfaces between tiles and heat sink. Thanks to these activities, the on-going production of the target elements is successful and allowed the fabrication of the target modules to be started. A reliable high heat flux divertor allowing the long pulse operation of $\mathrm{W} 7-\mathrm{X}$ is required to experimentally demonstrate and confirm the potential of the Helias stellarator line [19].

\section{ACKNOWLEDGMENT}

Pre-series phase II have been performed under the Grant Nr. 03FUS0014A from the Federal Ministry of Education and Research. Only the authors are responsible for the content of this publication.

\section{REFERENCES}

[1] T. Klinger et al., "Towards Assembly Completion and Preparation of Experimental Campaigns of Wendelstein 7-X in the perspective of a path to a Stellarator Fusion Plant”, Fus. Eng. Design, "in press”.

[2] T. Bräuer, T. Klinger, and H. S. Bosch, „Progress, Challenges, and Lessons Learned in the Construction of Wendelstein 7-X“, IEEE Trans. Plasma Sci., vol. 40, no. 3, pp. 577-583, March 2012.

[3] H.-S. Bosch, A. Dinklage, T. Klinger, and R. Wolf, "Physics programme for initial operation of Wendelstein 7-X”, Cont. Plasma Phys., vol. 50, no. 8, pp. 687-694, 2010.

[4] A. Peacock et al., "Progress in the design and development of a test divertor (TDU) for the start of W7-X operation”, Fus. Eng. and Des., vol. 84, no. 7-11, pp. 1475-1478, June 2009.

[5] A. Peacock et al., "Wendelstein 7-X High Heat Flux Components", this conference.

[6] M. Lipa, J. Schlosser, and F. Escourbiac, "Development of Actively Cooled Plasma Facing Components for Tore Supra”, Fus. Sci. Techn., vol. 56, no. 3, pp. 1124-1149, October 2009.

[7] M. Missirlian et al., "Influence of CFC Quality on the Performance of TS limiter elements under cyclic heat loading”, Fus. Eng. Des., vol. 86, no. 9-11, pp. 1579-1582, October 2011.

[8] G. Pintsuk et al., "Mechanical and thermo-physical characterization of three-directional carbon fiber composites for W-7X and ITER", Fus. Eng. Des., vol. 84, no. 7-11, pp. 1525-1530, June 2009.

[9] M. Merola, W. Dänner, J. Palmer, G. Vieider, and C. H. Wu, “ European contribution to the development of the ITER divertor", Fus. Eng. Des., vol. 66-68, pp. 211-217, September 2003.

[10] J. Boscary et al., "Pre-series and testing route for the serial fabrication of W7-X target elements”, Fus. Eng. Des., vol. 84, no. 26, pp. 497-500, June 2009.

[11] A. Plankensteiner, A. Leuprecht, B. Schedler, K.-H. Scheiber, and H. Greuner, "Finite element based design optimization of Wendelstein 7-X divertor components under high heat flux loading”, Fus. Eng. Des., vol. 82, no. 15-24, pp. 1813-1819, October 2007.

[12] H. Greuner et al., "Review of the high heat flux testing as an integrated part of W7-X divertor development”, Fus. Eng. Des., vol. 84, no. 2-6, pp. 848-852, June 2009. 
[13] H. Greuner et al., "Cyclic heat load testing of improved $\mathrm{CFC} / \mathrm{Cu}$ bonding for the W 7-X divertor targets”, Fus. Eng. Des., vol. 386388, no. 30, pp. 772-775, April 2009.

[14] J. Boscary et al., "Results of the examinations of the W7-X preseries target elements”, Fus. Eng. Des., vol. 82, no. 15-24, pp. 1634 1638 October 2007.

[15] H. Greuner, B. Böswirth, J. Boscary, and P. McNeely, "High heat flux facility GLADIS: operational characteristics and results of W7X pre-series target tests”, J. Nucl. Mat., Vol. 367-370, Part B, pp. 1444-1448, August 2007.

[16] M. Smirnow et al., "Development of a thermo-hydraulic bypass leakage test method for the Wendelstein 7-X target element cooling structure”, Fus. Eng. Des., vol. 86, no. 9-11, pp. 1732-1735, October 2011.

[17] J. Boscary et al., "Design improvement of the target elements of Wendelstein 7-X divertor”, Fus. Eng. Des., vol. 87, no. 7-8, pp. 1453-1456, August 2012.

[18] H. Traxler, P. Schuler, "Pulsed thermography inspection of the target elements for the W7-X divertor”, Phys. Scripta, T128, pp. 242-245, March 2007.

[19] F. Schauer, K. Egorov, and V. Bykov, "HELIAS 5-B magnet system structure and maintenance concept", "in press". 